\title{
An Assessment of the Level of Knowledge and Compliance with Infection Prevention and Control Standards Among Nurses in the Northern Regional Hospital
}

\author{
Shamsu-Deen Ziblim ${ }^{1,}$, , Sufyan Bakuri Suara ${ }^{1}$, Mohammed Mutaru Tahiru² \\ ${ }^{1}$ Department of Community Health and Family Medicine, School of Medicine and Health Science, University for Development Studies, \\ Tamale, Ghana \\ ${ }^{2}$ Northern Regional Health Directorate, Ghana Health Service, Tamale, Ghana
}

Email address:

zshamsu72@gmail.com (Shamsu-Deen Z.), suara.ghana@yahoo.com (Sufyan B. S.), nyagsimplus@gmail.com (Mohammed M. T.)

${ }^{*}$ Corresponding author

\section{To cite this article:}

Shamsu-Deen Ziblim, Sufyan Bakuri Suara, Mohammed Mutaru Tahiru. An Assessment of the Level of Knowledge and Compliance with Infection Prevention and Control Standards Among Nurses in the Northern Regional Hospital. World Journal of Public Health. Vol. 5, No. 4, 2020, pp. 84-88. doi: 10.11648/j.wjph.20200504.12

Received: October 18, 2020; Accepted: October 30, 2020; Published: November 9, 2020

\begin{abstract}
Background: Nosocomial infections are associated with a high risk of morbidity and mortality among hospitalized clients. They also predispose health care workers to an increased risk of infections. However, information on knowledge of infection prevention and control is scarce in Northern Ghana. Therefore, the objective of the study was to assess the knowledge and compliance of infection prevention and control practices among nurses at the Northern Regional Hospital Tamale, Ghana. Methods: The study was cross-sectional in design. Also, the data were gathered from randomly selected 268 nurses. In collecting the data, self-administered questionnaires were used. The data were analyzed using IBM SPSS V. 21. Results: From the results, the majority $(60.5 \%)$ of the respondents had high IPC knowledge, $25.8 \%$ had moderate IPC knowledge level, and only $13.8 \%$ had low IPC knowledge level. The findings on IPC compliance showed that the majority (77.6\%) of the respondents had a low IPC compliance level, $19.8 \%$ had a moderate IPC compliance level, and only $2.6 \%$ had a high IPC compliance level. Conclusion: In conclusion, most of the respondents had good knowledge of the IPC guidelines in the hospital. The present results may be useful in the formulation of policies for improved infection prevention at health facilities. We recommend that hospital authorities should intensify compliance with IPC guidelines.
\end{abstract}

Keywords: Infections, Control, Nosocomial, Compliance, Mortality, Morbidity

\section{Introduction}

Nosocomial infections are a big worry to leaders in the health care system, particularly in developing countries [1]. Compared with the developed countries, there is a higher risk of nosocomial infections in developing countries [2]. Health care workers spend the majority of their time with their clients. Therefore, they should possess knowledge of infection prevention and control practices (IPC) and comply with them to minimize the risk of infections.

However, in many developing countries, previous studies among healthcare staff showed low knowledge and compliance level of infection prevention and control practices [3-5]. For example, in Zimbabwe, the majority of the nurses $(72 \%)$ were said to lack knowledge on infection control principles, while $42 \%$ of the nurses also did not comply with infection control standards [3]. Moreover, in Ghana, a previous study showed that only $38.9 \%$ of the nurses recapped used needles before disposal [4]. However, this earlier study did not evaluate the level of knowledge of standard safety practices. Meanwhile, knowledge of health safety standard protocols is a key determinant of the compliance level of such protocols $[6,7]$. Therefore, to minimize the risk of nosocomial infections, it is vital to understand the level of knowledge of health staff on standard safety protocols and the compliance level of such standards.

Inadequate IPC related practices increase the risk of infections among health care workers and their clients [8-10]. 
Furthermore, $62 \%$ and $31 \%$ of all mortalities in Africa, and Southeast Asia, respectively, are linked with infectious disease [11]. Thus, nosocomial infections put an extra burden on both patients and the health care staff [12].

Nurses play a significant role in maintaining effective disease inhibition and control. However, their activities may also lead to the spread of infection [13]. Hence, the establishment of the IPC protocol aims to protect both the public and the health care staff from contracting an infection at a point of seeking health service and health care provision, respectively. Hence, the infections can be reduced by the provision of adequate knowledge of IPC protocols [5, 14]. Therefore, the Ministry of Health and the Ghana Health Services developed a policy framework on IPC 2009 with the ultimate goal of improving the capacity of healthcare workers in preventing and controlling infections and maintaining safety in healthcare settings [15].

In Ghana, among health care workers, previous authors have attempted to investigate their knowledge of nosocomial infections as well as their attitudes toward standard prevention and control practices. However, those studies were conducted in the Southern part of the country [16-19], which creates a knowledge gap in the Northern zone.

Thus, health policymakers may not be aware of the knowledge and compliance level of safety protocols of infection prevention and control among health care staff in the region. Hence, the objective of this study was to assess the knowledge and compliance level of infection prevention and control among nurses in the Northern regional hospital.

\section{Methods}

The study adopted a facility-based descriptive crosssectional study. We randomly recruited 268 nurses from Tamale Central Hospital, Ghana. Data were collected on socio-demographic variables, knowledge of IPC, and practices of IPC, using a structured questionnaire. Ethical approval of the study was obtained from the Internal Review Board (IRB) of the University for Development Studies (UDS) and the Tamale Central Hospital. Moreover, permission was obtained from the various Heads of departments in the hospital before data collection. Participating in this study was voluntary. Also, informed consent of participants was obtained from the study participants with the assurance of data confidentiality. The Statistical Package for Social Sciences (IBM SPSS v. 21 on window 10) was used for the data analysis. The results were presented as descriptive statistics.

\section{Results}

Table 1 below shows the demographic characteristics of the respondents. The majority $(54.5 \%)$ of the respondents were within the ages (years) of $21-30$. With the sex of the study subjects, the majority $(69.8 \%)$ of the respondents were females. Also, most (48.1\%) of the subjects were general nurses, and the majority $(76.9 \%)$ of them had the highest college education.

Table 1. Demographic characteristics of respondents.

\begin{tabular}{|c|c|c|c|}
\hline & & Frequency $(n=268)$ & Percentage \\
\hline \multirow{4}{*}{ Age group (years) } & $21-30$ & 146 & $54.5 \%$ \\
\hline & $31-40$ & 109 & $40.7 \%$ \\
\hline & $41-50$ & 9 & $3.4 \%$ \\
\hline & $51-59$ & 4 & $1.5 \%$ \\
\hline \multirow{2}{*}{ Sex of the respondent } & Male & 81 & $30.2 \%$ \\
\hline & Female & 187 & $69.8 \%$ \\
\hline Highest level of education/training & University & 62 & $23.1 \%$ \\
\hline
\end{tabular}

Table 2 below shows the respondents' IPC knowledge variables. In this study, most $(94.0 \%)$ of the respondents knew that hospital-acquired infections can be transmitted through medical equipment. Moreover, many (206) of them disagreed that nosocomial infections are infection from home. Also, about $65.3 \%$ of them agreed to know WHO five moments of hand hygiene. Moreover, $90.3 \%$ of the respondents agreed that standard safety measures apply to all patients and $87.3 \%$ of them knew that all staff and patients should be considered vulnerable to infections. With glove use, $89.6 \%$ of them knew that one cannot handle body fluids with bare hands if gloves are not available.

Table 2. Respondents IPC knowledge variables.

\begin{tabular}{|c|c|c|c|}
\hline Variable & & Frequency $(n=268)$ & Percentage \\
\hline \multirow{3}{*}{$\begin{array}{l}\text { Hospital-acquired infections (HAIs) may possibly be transmitted by medical } \\
\text { equipment. }\end{array}$} & True & 252 & $94.0 \%$ \\
\hline & False & 16 & $6.0 \%$ \\
\hline & Not applicable & 0 & $0.0 \%$ \\
\hline \multirow{3}{*}{ Nosocomial infections are brought from the house to the hospital by patients } & True & 56 & $20.9 \%$ \\
\hline & False & 206 & $76.9 \%$ \\
\hline & Not applicable & 6 & $2.2 \%$ \\
\hline \multirow{2}{*}{$\begin{array}{l}\text { Respondent knows the } 5 \text { moments of hand hygiene proposed by World Health } \\
\text { Organization }\end{array}$} & True & 175 & $65.3 \%$ \\
\hline & Not applicable & 28 & $10.4 \%$ \\
\hline \multirow{3}{*}{ Know that some equipment can be stored in antiseptic solutions for about 36 hours } & True & 52 & $19.4 \%$ \\
\hline & False & 202 & $75.4 \%$ \\
\hline & Not applicable & 14 & $5.2 \%$ \\
\hline
\end{tabular}




\begin{tabular}{|c|c|c|c|}
\hline Variable & & Frequency $(n=268)$ & Percentage \\
\hline \multirow{3}{*}{$\begin{array}{l}\text { In beds shortages, patients with communicable disease can be admitted in the } \\
\text { same ward with others with noncommunicable diseases }\end{array}$} & True & 50 & $18.7 \%$ \\
\hline & False & 202 & $75.4 \%$ \\
\hline & Not applicable & 16 & $6.0 \%$ \\
\hline \multirow{3}{*}{ In using clean water, micro-organisms are destroyed } & True & 54 & $20.1 \%$ \\
\hline & False & 211 & $78.7 \%$ \\
\hline & Not applicable & 3 & $1.1 \%$ \\
\hline \multirow[t]{3}{*}{ I know that safety precautions should be applied to all patients } & True & 242 & $90.3 \%$ \\
\hline & False & 19 & $7.1 \%$ \\
\hline & Not applicable & 7 & $2.6 \%$ \\
\hline \multirow[t]{3}{*}{ Familiar with hospital-acquired infection standards. } & True & 212 & $79.1 \%$ \\
\hline & False & 46 & $17.2 \%$ \\
\hline & Not applicable & 10 & $3.7 \%$ \\
\hline \multirow[t]{3}{*}{ Know that all staff, and patients should be considered potentially infectious } & True & 234 & $87.3 \%$ \\
\hline & False & 33 & $12.3 \%$ \\
\hline & Not applicable & 1 & $0.4 \%$ \\
\hline \multirow{3}{*}{ If gloves are unavailable, I can handle body fluids with bare hands. } & True & 19 & $7.1 \%$ \\
\hline & False & 240 & $89.6 \%$ \\
\hline & Not applicable & 9 & $3.4 \%$ \\
\hline \multirow[t]{4}{*}{ Used needles can be recapped after use and before their disposal } & True & 107 & $39.9 \%$ \\
\hline & False & 154 & $57.5 \%$ \\
\hline & Not applicable & 7 & $2.6 \%$ \\
\hline & Not applicable & 7 & $2.6 \%$ \\
\hline
\end{tabular}

Table 3 below indicates the IPC compliance variables among the study respondents. The Hand hygiene was practiced by majority $(98.9 \%)$ of the respondents before and after patient contact. Additionally, most ( $81.0 \%)$ of them disagreed with the shaking of linen to release dust. Moreover, only $51.1 \%$ of the respondents had access to guidelines/Training manuals in their unit. Also, about $58.2 \%$ of them always put on a mask and glasses when performing invasive and body fluid procedures. Besides, about $58.2 \%$ of the hospital staff always put on nose mask and glasses when performing invasive and body fluid procedures. Concerning the shaking of linen, the qualitative data equally supported the quantitative data.

Table 3. Respondents IPC compliance variables.

\begin{tabular}{|c|c|c|c|}
\hline Variable & & Frequency $(n=268)$ & Percentage \\
\hline \multirow{3}{*}{ I have always washed my hands before, and after a contact with patients. } & Agree & 265 & $98.9 \%$ \\
\hline & Disagree & 3 & $1.1 \%$ \\
\hline & Not applicable & 0 & $0.0 \%$ \\
\hline \multirow{3}{*}{$\begin{array}{l}\text { When I am to perform an invasive and body fluid procedure, I put on a nose mask } \\
\text { and glasses }\end{array}$} & Agree & 156 & $58.2 \%$ \\
\hline & Disagree & 91 & $34.0 \%$ \\
\hline & Not applicable & 21 & $7.8 \%$ \\
\hline \multirow{2}{*}{ In this hospital, the leaders monitor knowledge of infection prevention and control } & Agree & 151 & $56.3 \%$ \\
\hline & Not applicable & 10 & $3.7 \%$ \\
\hline \multirow{3}{*}{ We have access to IPC Guidelines/Training manuals in our unit } & Agree & 154 & $57.5 \%$ \\
\hline & Disagree & 105 & $39.2 \%$ \\
\hline & Not applicable & 9 & $3.4 \%$ \\
\hline \multirow{3}{*}{ Our hospital has IPC committee in place to monitor IPC activities } & Agree & 137 & $51.1 \%$ \\
\hline & Disagree & 105 & $39.2 \%$ \\
\hline & Not applicable & 26 & $9.7 \%$ \\
\hline \multirow{2}{*}{$\begin{array}{l}\text { Screening of patients are being done to detect colonization even if no evidence of } \\
\text { infection }\end{array}$} & Disagree & 154 & $57.5 \%$ \\
\hline & Not applicable & 13 & $4.9 \%$ \\
\hline \multirow{3}{*}{ Vaccination is provided to staff } & Agree & 32 & $11.9 \%$ \\
\hline & Disagree & 213 & $79.5 \%$ \\
\hline & Not applicable & 23 & $8.6 \%$ \\
\hline \multirow{3}{*}{ We wear PPE when handling linens } & Agree & 123 & $45.9 \%$ \\
\hline & Disagree & 126 & $47.0 \%$ \\
\hline & Not applicable & 19 & $7.1 \%$ \\
\hline \multirow{3}{*}{ Linens are shaking out to release dust from the linen } & Agree & 21 & $7.8 \%$ \\
\hline & Disagree & 217 & $81.0 \%$ \\
\hline & Not applicable & 30 & $11.2 \%$ \\
\hline
\end{tabular}

\section{Discussion}

In this study, the knowledge level and practices of IPC were assessed among nurses in Tamale Regional Hospital.
The IPC compliance is influenced by knowledge of IPC components. For instance, hand hygiene, use of personal protective equipment, sterilization, disinfection, vaccination, active surveillance of suspected infections, outbreak investigation, and management [20]. Regarding the 
knowledge level and practices of IPC, in the present study, most $(94.0 \%)$ of the respondents knew that HAIs can be transmitted through medical equipment (syringes, needles, catheters, stethoscope, and thermometers). Moreover, about $65.3 \%$ of the respondents agreed to know standard hand hygiene practices. Moreover, about $65.3 \%$ of the respondents agreed to know standard hand hygiene practices. Similarly, in an earlier study by Alrubaiee et al [21] in Yemen, most of the participants $(72.9 \%)$ possessed fair level of knowledge on hand hygiene practices with about $35.3 \%$ also possessing a good level of knowledge on personal protective equipment. Also, in that study, $67.1 \%$ had a poor level of knowledge concerning safe injection practices. In contrast, in Kenya, a previous study found low standard precaution practice including, hand hygiene [22].

In this study, about $90.3 \%$ of the respondents agreed they apply standard precautions to all patients regardless of their diagnosis, and $87.3 \%$ of them knew that all staff and patients are vulnerable to infections. With glove use, $89.6 \%$ of them knew that one cannot handle body fluids with bare hands if gloves are not available. Also, the majority (60.5\%) of the respondents had high IPC knowledge, $25.8 \%$ had moderate IPC knowledge level, and $13.8 \%$ had low IPC knowledge level. In line with the present observations, in a previous study in Nigeria among nurses, showed that $66 \%$ of them showed good knowledge of IPC [23]. Also, a similar study in Ghana by Ocran et al. [24] in the Central Regional Hospital showed that about $88.7 \%$ of the healthcare workers were well-informed on HAIs. Conversely, an earlier crosssectional study conducted by Geberemariyam et al. [25] in Ethiopia found that about $53.7 \%$ of the participants were knowledgeable on IPCs.

With compliance with safety protocols, hand hygiene was practiced by the majority ( $98.9 \%$ ) before and after patient contact. The present results corroborate with the findings of Russell et al. [26] study, where there was high adherence to numerous IPC practices, such as appropriate hand hygiene, before and after patient care activities (99.4\%). However, another study by Geberemariyam et al. [25] indicated that $69.4 \%$ (450) indicated that they regularly wash their hands after attending to a patient, $56.1 \%$ (364) before patient care, 64.2\% (416) after removing gloves and $63.6 \%$ (412) before care of wounds. Appropriate hand washing before and after patient contact may reduce the risk of HAIs to about $6 \%$, and about $14 \%$ risk reduction in Clostridium difficile infection [27].

In the current study, it was observed that about $58.2 \%$ of the respondents always put on a mask and glasses anytime they are performing an invasive or body fluid procedures. This observation is low when compared to a previous study by Russell et al. [26], in which $69.6 \%$ of the respondents adhered to IPC practices relating to wearing of goggles or eye shields when exposed to bodily fluids. However, another study found lower proportions of adherence to IPC standards when compared with the present results. In that study by Hayeh et al.[5], the total level of compliance with IPC guidelines was about $45.1 \%$. Moreover, the authors found that about $58.2 \%$ of the hospital staff always put on a mask and glasses in their line of duty.
Meanwhile, low level of practices and adherence to IPC standards have been associated with the increasing rates of HAIs especially in developing countries such as Ethiopia [25]. The IPC practices are an important factor in the health care system and is implicated in key areas including epidemiology, infectious diseases, health system strengthening as well as social sciences. The IPC compliance may protect not only patients but the healthcare workers and the general public from the risk of contracting infections from the healthcare settings [28].

\section{Conclusion}

The majority $(60.5 \%)$ of the participants in the study reported a high IPC knowledge. Also, the compliance level of IPC practices was high $(77.6 \%)$. Nonetheless, we recommend that education on the standard practices of infection prevention and control at health facilities be intensified among health staff in Northern Regional Hospital, Ghana to improve

\section{Conflict of Interest}

All the authors do not have any possible conflicts of interest.

\section{Acknowledgements}

We are so thankful to Doctor Ibunwanya (Medical Director of Northern Regional Hospital Tamale) and Hajiah Ewuntomah, Yapaga Amina for assisting us organize our study participants.

\section{References}

[1] Mehtar S. Bridging the infection prevention and control (IPC) gap between theory and implementation - An African experience. Int $\mathrm{J}$ Infect Dis. 2014; 21: 52. doi: 10.1016/j.ijid.2014.03.528.

[2] Bello A, Emannuel BO, Adegoke B, Bello A. Nosocomial infections: knowledge and source of information among clinical health care students in Ghana. Int J Gen Med. 2011.

[3] Tirivanhu C. Barriers to infection prevention and control (IPC) practice among nurses at Bindura Provincial Hospital, Zimbabwe. IOSR J Nurs Heal Sci. 2014.

[4] Konlan KD, Aarah-Bapuah M, Kombat JM, Wuffele GM. The level of nurses' knowledge on occupational post exposure to hepatitis B infection in the Tamale metropolis, Ghana. BMC Health Serv Res. 2017; 17: 254. doi: 10.1186/s12913-0172182-7.

[5] Hayeh PA, Kwasi Esena R. Infection Prevention and Control Practices among Health Workers at Ridge Regional Hospital in Accra Ghana. Int J Heal Sci Res. 2013; 3: 47.

[6] Oliveira AC, Cardoso CS, Mascarenhas D. Precauções de contato em Unidade de Terapia Intensiva: fatores facilitadores e dificultadores para adesão dos profissionais. Rev da Esc Enferm da USP. 2010; 44: 161-5. doi: 10.1590/S008062342010000100023 . 
[7] Mukamurenzi AE. Compliance with infection control standard precautions in prevention of surgical site infection among staff in operating room at a selected refferal hospital in Rwanda. 2019.

[8] Houghton C, Meskell P, Delaney H, Smalle M, Glenton C, Booth $\mathrm{A}$, et al. Barriers and facilitators to healthcare workers' adherence with infection prevention and control (IPC) guidelines for respiratory infectious diseases: a rapid qualitative evidence synthesis. Cochrane Database Syst Rev. 2020.

[9] Wilkason C, Lee C, Sauer LM, Nuzzo J, McClelland A. Assessing and Reducing Risk to Healthcare Workers in Outbreaks. Heal Secur. 2020; 18: 205-11.

[10] Jacob JT, Herwaldt LA, Durso FT, Program CDCPE. Preventing healthcare-associated infections through human factors engineering. Curr Opin Infect Dis. 2018; 31: 353-8.

[11] Curtis VA, Danquah LO, Aunger R V. Planned, motivated and habitual hygiene behaviour: an eleven country review. Health Educ Res. 2009; 24: 655-73. doi: 10.1093/her/cyp002.

[12] Bobadilla J-L, Cowley P, Musgrove PA, Saxenian H. The essential package of health services in developing countries. World Dev report Washington, DC World Bank. 1994.

[13] Page K, Wilson M, Parkin IP. Antimicrobial surfaces and their potential in reducing the role of the inanimate environment in the incidence of hospital-acquired infections. J Mater Chem. 2009; 19: 3819-31.

[14] McDonald LC, Gerding DN, Johnson S, Bakken JS, Carroll $\mathrm{KC}$, Coffin SE, et al. Clinical practice guidelines for Clostridium difficile infection in adults and children: 2017 update by the Infectious Diseases Society of America (IDSA) and Society for Healthcare Epidemiology of America (SHEA). Clin Infect Dis. 2018; 66: e1-48.

[15] Ministry of Health. Policy and Guidelines for Infection Prevention and Control in Healthcare facilities. MOH/GHS. Accra; 2009.

[16] Hesse A, Adu-Aryee N, Entsua-Mensah K, Wu L. Knowledge, attitude and practice universal basic precautions by medical personnel in a Teaching Hospital. Ghana Med J. 2006.

[17] Amponsah-Tawaih K, Adu MA. Work Pressure and Safety Behaviors among Health Workers in Ghana: The Moderating Role of Management Commitment to Safety. Saf Health Work. 2016; 7: 340-6. doi: 10.1016/j.shaw.2016.05.001.
[18] Akagbo SE, Nortey P, Ackumey MM. Knowledge of standard precautions and barriers to compliance among healthcare workers in the Lower Manya Krobo District, Ghana. BMC Res Notes. 2017; 10: 432. doi: 10.1186/s13104-017-2748-9.

[19] Tagoe D, Desbordes K. Investigating potential sources of transmission of healthcare-associated infections in a regional hospital, Ghana. Int J Appl Basic Med Res. 2012.

[20] Ministry of Health. National Policy and guidelines for infection prevention and control in healthcare settings. Accra, Ghana. Accra; 2015.

[21] Alrubaiee G, Baharom A, Shahar HK, Daud SM, Basaleem HO. Knowledge and practices of nurses regarding nosocomial infection control measures in private hospitals in Sana'a City, Yemen. Saf Heal. 2017; 3: 1-6.

[22] Gertrude M. PREVENTION STANDARD PRECAUTIONS AMONG NURSES. 2013.

[23] Nwadike RN. International Journal of Basic, Applied and Innovative Research knowledge and practice of post-operative wound infection prevention among nurses in the surgical unit of a teaching. $2014 ; 3: 23-8$.

[24] Ocran I, Tagoe DNA. Knowledge and attitude of healthcare workers and patients on healthcare associated infections in a regional hospital in Ghana. Asian Pacific J Trop Dis. 2014; 4: 135-9. doi: 10.1016/S2222-1808 (14)60330-3.

[25] Geberemariyam BS, Donka GM, Wordofa B. Assessment of knowledge and practices of healthcare workers towards infection prevention and associated factors in healthcare facilities of West Arsi District, Southeast Ethiopia: A facilitybased cross-sectional study. Arch Public Heal. 2018.

[26] Russell D, Dowding DW, McDonald M V., Adams V, Rosati RJ, Larson EL, et al. Factors for compliance with infection control practices in home healthcare: findings from a survey of nurses' knowledge and attitudes toward infection control. Am J Infect Control. 2018; 46: 1211-7. doi: 10.1016/j.ajic.2018.05.005.

[27] Sickbert-Bennett EE, Dibiase LM, Schade Willis TM, Wolak ES, Weber DJ, Rutala WA. Reduction of healthcare-associated infections by exceeding high compliance with hand hygiene practices. Emerg Infect Dis. 2016.

[28] World Health Organization. Report on the Burden of Endemic Health Care-Associated Infection Worldwide Clean Care is Safer Care. 2018. 